\title{
GEM detector performance with innovative micro-TPC readout in high magnetic field
}

\author{
I. $\operatorname{Garzia}^{(1)(2)}$, M. Alexeev ${ }^{(3)}$, A. Amoroso ${ }^{(3)(4)}$, R. Baldini Ferroli(5)(6), M. Bertani ${ }^{(5)}$, D. Bettoni(1), \\ F. Bianchi ${ }^{(3)(4)}$, A. Calcaterra ${ }^{(5)}$, N. Canale ${ }^{(2)}$, M. Capodiferro ${ }^{(5)(7)}$, V. Cassariti ${ }^{(1)}$, S. Cerioni $^{(5)}$,

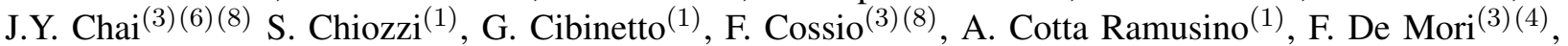 \\ M. Destefanis ${ }^{(3)(4)}$, J. Dong( ${ }^{(5)}$, F. Evangelisti $^{(1)}$, R. Farinelli( ${ }^{(1)}(2)$, L. Fava $^{(3)}$, G. Felici $^{(5)}$, E. Fioravanti ${ }^{(1)}$,

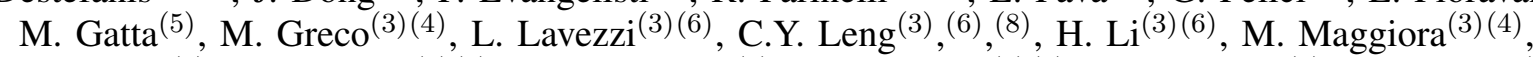 \\ R. Malaguti ${ }^{(1)}$, S. Marcello ${ }^{(3)(4)}$, M. Melchiorri(1) ${ }^{(1)}$ G. Mezzadri ${ }^{(1)(2)}$, M. Mignone $^{(3)}$, G. Morello $^{(5)}$,

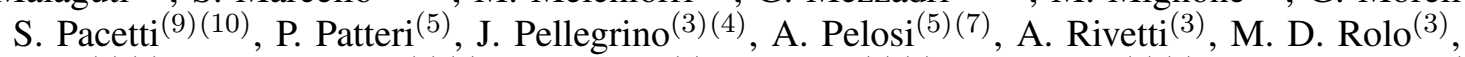 \\ M. Savrié(1)(2), M. Scodeggio ${ }^{(1)(2)}$, E. Soldani ${ }^{(5)}$, S. Sosio ${ }^{(3)(4)}$, S. Spataro ${ }^{(3)(4)}$, E. Tskhadadze ${ }^{(5)(11)}$, \\ S. Verma ${ }^{(2)}$, R. Wheadon ${ }^{(3)}$, L. Yan ${ }^{(3)}$ \\ ${ }^{(1)}$ INFN - Sezione di Ferrara - via G. Saragat 1, 44122 Ferrara, Italy \\ ${ }^{(2)}$ Università di Ferrara, Dipartimento di Fisica e Scienze della Terra - via G. Saragat 1, 44122 Ferrara, Italy \\ ${ }^{(3)}$ INFN - Sezione di Torino - via P. Giuria 1, 10125 Torino, Italy \\ ${ }^{(4)}$ Università di Torino - via P. Giuria 1, 10125 Torino, Italy \\ (5) INFN - Laboratori Nazionale di Frascati - via E. Fermi 40, 00044 Frascati (Roma), Italy \\ ${ }^{(6)}$ Institute of High Energy Physics, Chinese Academy of Sciences, 19B YuquanLu, Beijing, 100049, China \\ (7) INFN - Sezione di Roma, c/o Università la Sapienza, p.le Aldo Moro 2, 00185 Roma, Italy \\ (8) Politecnico di Torino, Dipartimento di Elettronica e Telecomunicazioni, C.so Duca degli Abruzzi 24, 10129 Torino, Italy \\ ${ }^{(9)}$ INFN - Sezione di Perugia, via A. Pascoli 14, 06123 Perugia, Italy \\ ${ }^{(10)}$ Università di Perugia, Dipartimento di Fisica e Geologia, via A. Pascoli 14, 06123 Perugia, Italy \\ (11) Joint Institute for Nuclear Research (JINR), Joliot-Curie 6, Dubna, Moscow region, 141980, Russia
}

\begin{abstract}
Gas detector development is one of the pillars of the research in fundamental physics. Since several years, a new concept of detectors, called Micro Pattern Gas Detector (MPGD), allowed to overcome several problems related to other types of commonly used detectors, like drift chamber and micro strips detectors, reducing the rate of discharges and providing better radiation tolerance. Among the most used MPGDs are the Gas Electron Multipliers (GEMs). Invented by Sauli in 1997, nowadays GEMs have become an important reality for particle detectors in high energy physics. Commonly deployed as fast timing detectors and triggers, their fast response, high rate capability and high radiation hardness make them also suitable as tracking detectors. The readout scheme is one of the most important features in tracking technology. Analog readout based on the calculation of the center of gravity technique allows to overcome the limit imposed by digital pads, whose spatial resolution is limited by the pitch dimensions. However, the presence of high external magnetic fields can distort the electronic cloud and affect the performance. The development of the micro-TPC reconstruction method brings GEM detectors into a new prospective, improving significantly the spatial resolution in presence of high magnetic fields. This innovative technique allows to reconstruct the 3-dimensional particle position, as Time Projection Chamber, but within a drift gap of a few millimeters. In these report, the charge centroid and microTPC methods are described in details. We discuss the results of several test beams performed with planar chambers in magnetic field. These results are one of the first developments of microTPC technique for GEM detectors, which allows to reach unprecedented performance in a high magnetic field of $1 \mathrm{~T}$.
\end{abstract}

\section{INTRODUCTION}

The Gas Electron Multiplier (GEM) is a newly developed gaseous electron multiplier invented in 1997 by Fabio Sauli [1]. It consists of a thin insulating polymeric foil $(\sim 50$ $\mu \mathrm{m}$ of kapton) metal coated on both sides ( $3-5 \mu \mathrm{m}$ of copper), and pierced by a high density regular matrix of holes with a typical diameter of about $50 / 70 \mu \mathrm{m}$ [2] realized with a photolithographic method. The application of proper potential differences between cathode, two GEM electrode sides and anode generates a very intense dipole field in the holes. A charged track passing through the GEM detector ionizes the gas. The released electrons drift into the holes following the field lines, undergo avalanche multiplication and are collected on the anode. With a single GEM foil a gain up to $10^{3}$ can be achieved with moderate voltages. Better performance is obtained by means of three GEM (triple-GEM) foils between the cathode and the anode instead of one: gain values up to $10^{5}$ can be achieved working at lower voltages while reducing the discharge probability. Unless otherwise specified, all the results reported here are related to the triple-GEM configuration.

\section{TRACK RECONSTRUCTION METHODS}

GEM detectors can fulfil a large variety of requests thanks to their properties, such as the robustness, the possibility to build large area detectors, its relatively easy construction method with limited cost, the low aging effects and high reconstruction 


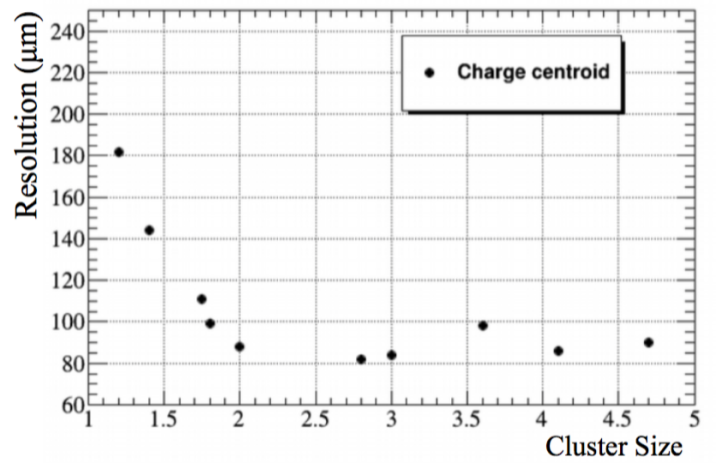

Fig. 1. Resolution from charge centroid method vs. cluster size for orthogonal tracks and without magnetic field. A planar triple-GEM with $5 \mathrm{~mm}$ drift gap filled with $\mathrm{ArCO}_{2}$ is used for this study.

efficiency, etc. However, the spatial resolution in presence of high magnetic fields is not optimal and new tracking reconstruction methods should be investigated in order to push the spatial resolution below $150 \mu \mathrm{m}$.

\section{A. Charge centroid method}

In the case of a Gaussian charge distribution on the anode, the position $x$ of a charged track relative to the anode can be determined by means of the weighted average of the fired strip positions $x_{i}$ :

$$
x=\frac{\sum_{i} x_{i} q_{i}}{\sum_{i} q_{i}}
$$

where the weights $q_{i}$ are defined by the charge collected by each fired strip. Figure 1 shows the spatial resolutions as a function of the average cluster size (or number of fired strips) measured under the condition of zero magnetic field and orthogonal tracks. A resolution of $80-90 \mu \mathrm{m}$ is achieved when the average cluster size is larger than two; for lower cluster size values, the performance of digital readout is limited by the strip pitch.

\section{MICRO-TPC METHOD}

When the charge distribution is not Gaussian, i.e. in presence of magnetic field or inclined tracks, the charge centroid method fails and a new reconstruction method should be investigated in order to keep a resolution of the order of $100 \mu \mathrm{m}$ : the micro-TPC $(\mu-$ TPC) method [3]. The drift gap (gap between the cathode and the first GEM foil) can be considered as a tiny time projection chamber: the time information related to each fired strip allows to perform a local track reconstruction in the few-mm drift gap. In Figure 2 is reported the drift time distribution for an event in which eight strips are hit and for which the charge distribution on the anode is no more Gaussian. Knowing the coordinate $x_{i}$ of each strip, the position of each primary ionization $z_{i}$ perpendicular to the strip plane is obtained from time measurement using the electron drift velocity from GARFIELD simulation. The $\left(x_{i}, z_{i}\right)$ points are then fitted with a linear function $z=a x+b$ and the position is extracted in the middle of the gap in order to minimize the errors [3] as

$$
x=\frac{g a p / 2-b}{a} .
$$

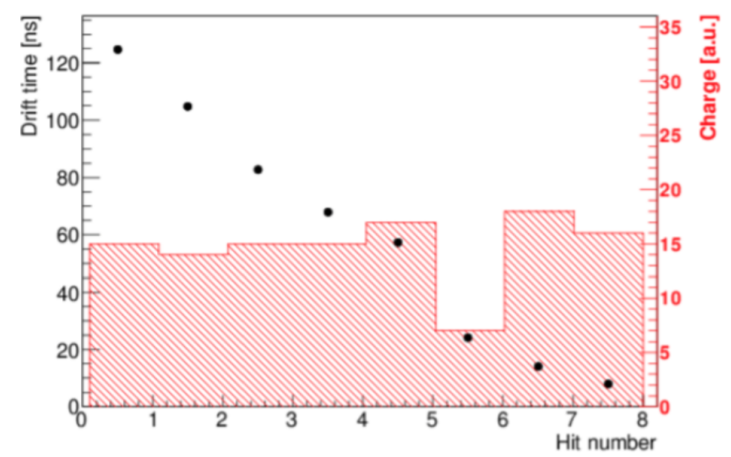

Fig. 2. Drift time distribution as a function of hit strips (points). The dashed histogram represents the charge distribution, which is no more Gaussian for this particular event.

\section{BEAM TEST RESULTS}

Planar chamber prototypes (see Fig. 3) have been tested on the H4 line of the SPS at CERN [4]. The chambers are $10 \times 10$ $\mathrm{cm}^{2}$ triple GEMs, with a bi-dimensional $X Y$ view readout and a drift gap of $3 \mathrm{~mm}$ or $5 \mathrm{~mm}$. Two different gas mixtures are used: $A r: \mathrm{CO}_{2}(70 / 30)$ and Ar:i- $\mathrm{C}_{4} \mathrm{H}_{10}(90 / 10)$. The main goal

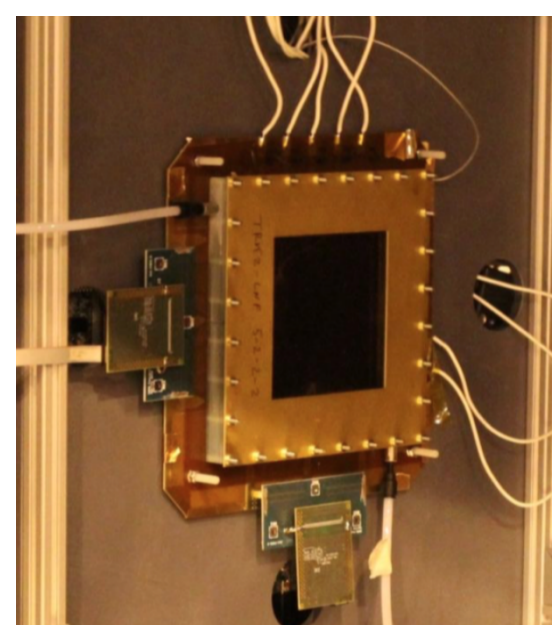

Fig. 3. Instrumented $10 \times 10 \mathrm{~cm}^{2}$ planar chamber used during beam test data taking.

of the beam test measurements is to study the performance as a function of several parameters, such as high voltage, field setting, gas mixture, drift gap, etc., in order to set the optimal working point needed to achieve the best efficiency and spatial resolution.

All the results reported below are obtained using a chamber with $5 \mathrm{~mm}$ drift gap. 

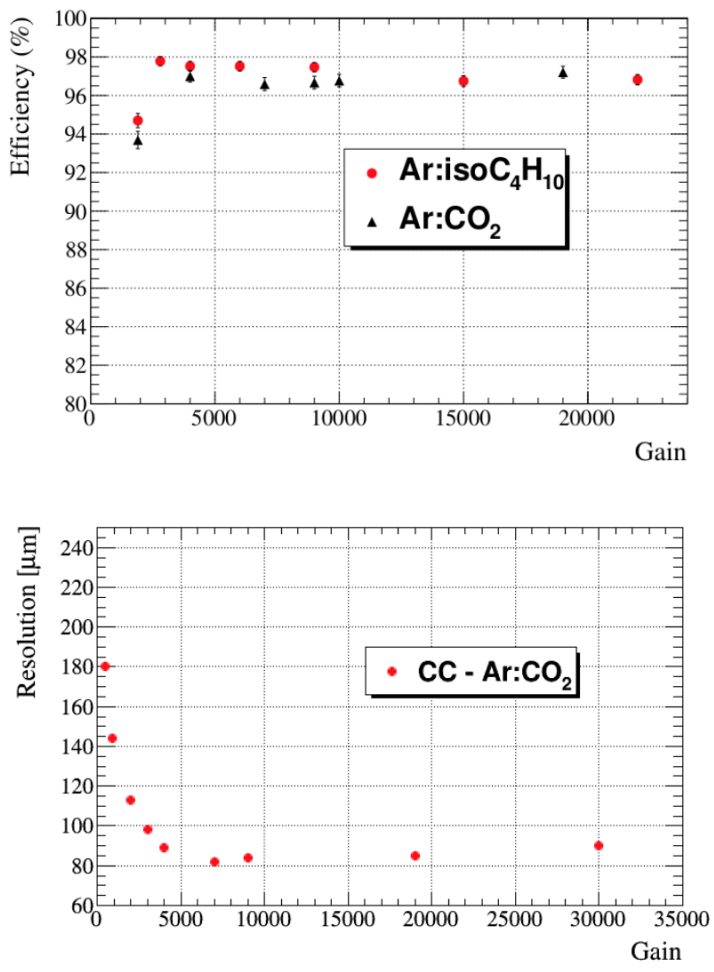

Fig. 4. Efficiency (upper plot) and resolution (lower plot) as a function of gain measured using the charge centroid method (CC) for orthogonal tracks without magnetic field.

\section{A. Orthogonal tracks without magnetic field}

The simplest case is for events with orthogonal tracks and no magnetic field, since the charge distribution on the anode is Gaussian and the charge centroid method works properly. Only the diffusion process and the avalanche multiplication apply. Charge centroid method is very accurate: we can reach a reconstruction efficiency of $97 \%-98 \%$ on the single $X / Y$ view and a spatial resolution $<100 \mu \mathrm{m}$ for a wide range of gain, as shown in Figure 4.

\section{B. Inclined tracks or/and magnetic field}

In most cases, charged tracks are not perpendicular to the GEM planes and in some high energy physics experiments they can be immersed in a magnetic field.

We can identify three different scenarios:

1) $B=0$ and inclined tracks: cluster size increases for increasing incident angles and the charge distribution on the anode becomes far from a Gaussian shape. The resolution measured with the charge centroid method worsens almost linearly with the angle, while the $\mu-\mathrm{TPC}$ method becomes more and more efficient, as shown in Figure 5a.

2) $B \neq 0$ and orthogonal tracks: the effect of the Lorentz force on the electrons must be taken into account. The magnetic field shifts the electrons avalanche with respect to the perpendicular path and enlarges the charge distribution on the anode, which leads to the deviation

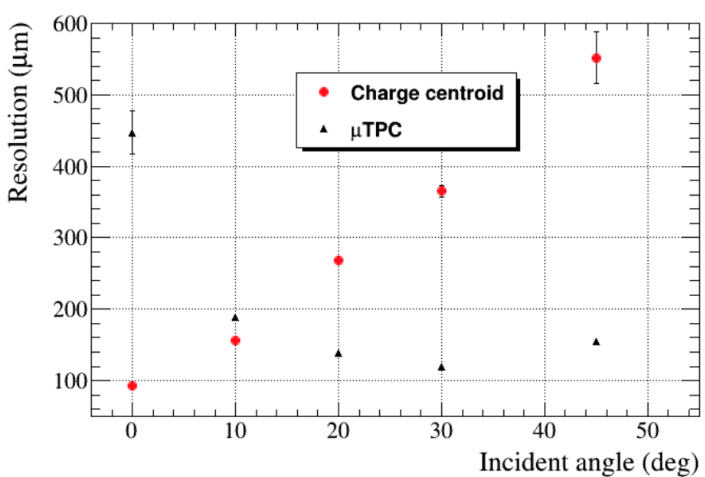

(a) $\mathrm{B}=0$ and inclined tracks.

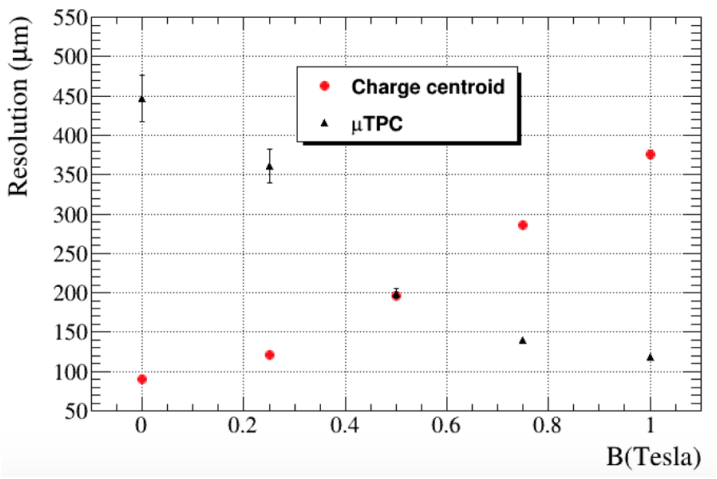

(b) $\mathrm{B} \neq 0$ and orthogonal tracks.

Fig. 5. Resolution measured using charge centroid and $\mu-$ TPC methods for two different scenarios: (a) inclined tracks with $\mathrm{B}=0$, and (b) $\mathrm{B} \neq 0$ and orthogonal tracks. These results are obtained with a gas mixture of Ar:i$\mathrm{C}_{4} \mathrm{H}_{10}$ and $5 \mathrm{~mm}$ drift gap. Points and triangles refer to charge centroid and $\mu-$ TPC results, respectively.

from a Gaussian shape. As shown in Fig. 5b, also in this case the charge centroid method deteriorates almost linearly for increasing magnetic field values, while the $\mu$ - TPC method reaches the best resolution value of about $120 \mu \mathrm{m}$ for $\mathrm{B}=1 \mathrm{~T}$. The parameter that describes the avalanche shift is the Lorentz angle, defined as the angle between the drift direction of the electrons under the influence of the magnetic field and the direction of the electric field and it must be used to correct the reconstructed track trajectory.

3) $B \neq 0$ and inclined tracks: this is the most interesting and awkward case. Two different effects should be taken into account in order to fully understand the beam test results. As depicted in Figure 6a, a focusing and defocusing effect appears, depending on the fact that the incident track and the Lorentz angles are concordant or discordant, respectively. The measured resolution for several incident angles in a magnetic field of $1 \mathrm{~T}$ is shown in Figure $6 \mathrm{~b}$. The charge centroid and $\mu-\mathrm{TPC}$ methods are complementary and their combination can provide a flat spatial resolution of $130 \mu \mathrm{m}$, which is the best resolution result obtained for a triple-GEM in a magnetic field of $1 \mathrm{~T}$. 


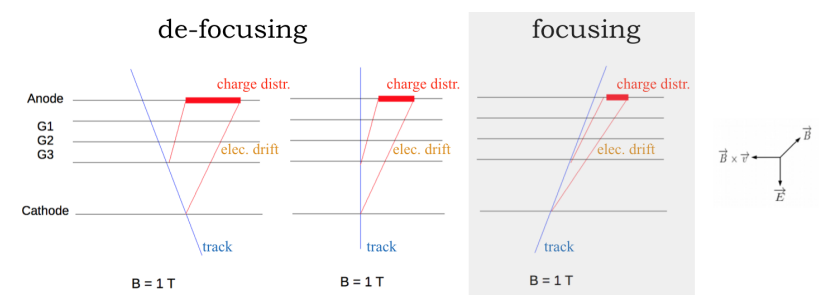

(a) De-focusing (white) and focusing (gray) effects.

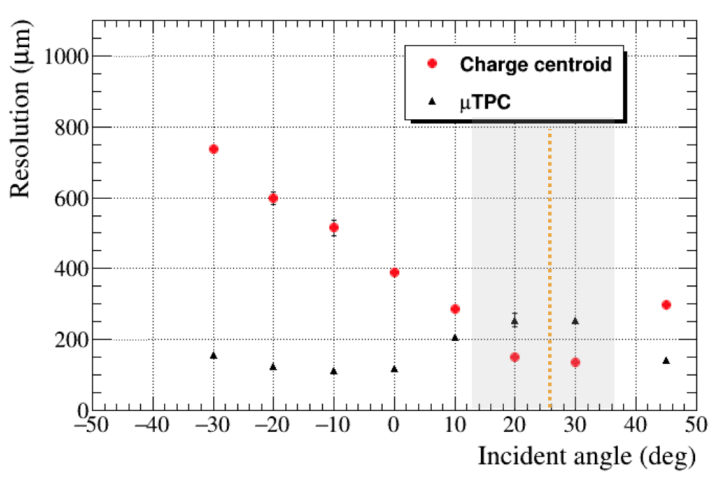

(b) Resolution vs. incident track angle for $\mathrm{B}=1$.

Fig. 6. (a) De-focusing and focusing effects for different configurations of incident angle. A de-focusing effect occurs when the Lorentz angle and the incident angle of the track are discordant, while a focusing effect (light gray highlighted area) when they are concordant. (b) Resolution vs. incident angle measured from a triple-GEM (Ar:i- $\mathrm{C}_{4} \mathrm{H}_{10}$ gas mixture and $5 \mathrm{~mm}$ drift gap) located in a magnetic field of $\mathrm{B}=1 \mathrm{~T}$. Focusing region is highlighted by the light gray area and the Lorentz angle $(\sim 26 \mathrm{deg})$ determined by the applied fields and the gas mixture is represented by the dashed orange line. Points and triangles refer to charge centroid and $\mu-\mathrm{TPC}$ results, respectively.

\section{V. $\mu-$ TPC OPTIMIZATION}

All the studies performed during the beam test are used to optimize the $\mu-\mathrm{TPC}$ performance: $\mathrm{Ar}$ : $\mathrm{i}-\mathrm{C}_{4} \mathrm{H}_{10}$ gas mixture provides higher electrical stability, an higher number of primary electrons and proper diffusion properties, while the $5 \mathrm{~mm}$ conversion gap gives the best resolution in magnetic field for inclined tracks. Another parameter that can be used to optimize the $\mu-$ TPC algorithm is the drift field.

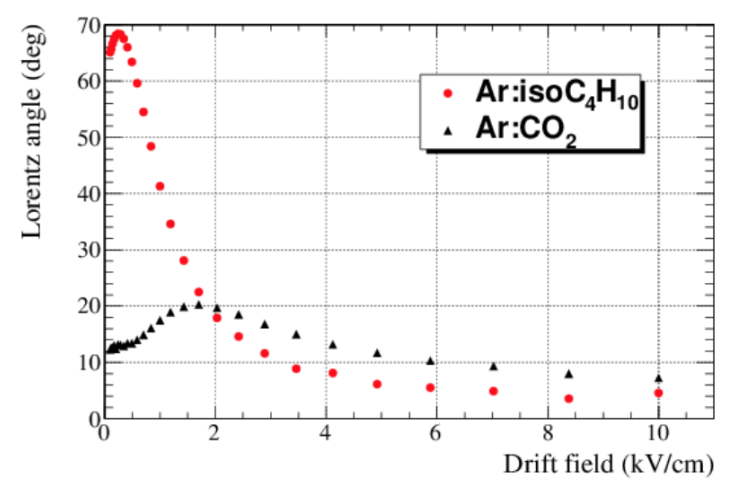

Fig. 7. GARFIELD simulation of Lorentz angle as a function of the drift field. For a gas mixture of Ar:i- $\mathrm{C}_{4} \mathrm{H}_{10}(90 / 10)$ and a drift field of $1.5 \mathrm{kV} / \mathrm{cm}$ the Lorentz angle is about $26 \mathrm{deg}$, in agreement with the behavior shown in Figure $6 b$.
The results shown in this report are obtained with the following electrical setting within the electrodes: $1.5 \mathrm{kV} / \mathrm{cm}$ in the drift gap, $3 \mathrm{kV} / \mathrm{cm}$ between the GEM foils, and $5 \mathrm{kV} / \mathrm{cm}$ between the last GEM foil and the anode.

From GARFIELD simulation, we can study the behavior of the Lorentz angle for different drift fields and, as shown in Figure 7, we observed a very strong dependence for drift field lower than $3 \mathrm{kV} / \mathrm{cm}$. Thus, the working region can be properly chosen by setting the drift field in such a way that the focusing region highlighted in Figure $6 \mathrm{~b}$ moves to the left or to the right depending on the experiment requirements.

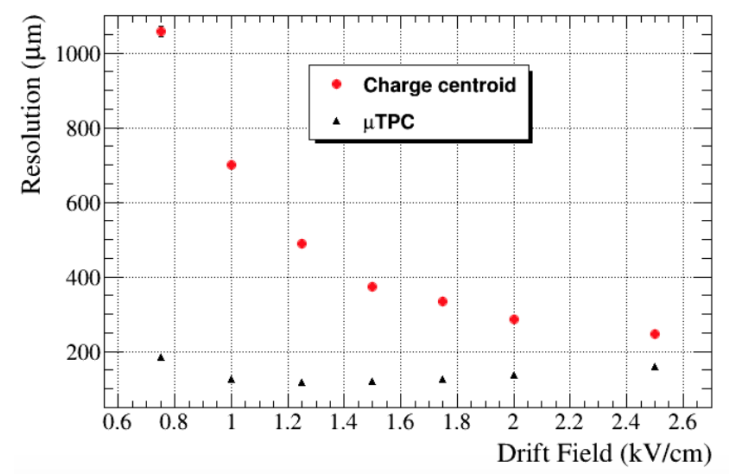

Fig. 8. Comparison of resolutions measured using charge centroid and $\mu-$ TPC method as a function of the drift field. These results are obtained using a gas mixture of Ar:i- $\mathrm{C}_{4} \mathrm{H}_{10}(90 / 10)$ and $5 \mathrm{~mm}$ drift gap. Points and triangles refer to charge centroid and $\mu-\mathrm{TPC}$ results, respectively.

Figure 8 shows the resolution measured using charge centroid and $\mu-\mathrm{TPC}$ methods for several configurations of drift fields up to $2.5 \mathrm{kV} / \mathrm{cm}$. For the charge centroid, we observe a worsening of the resolution for decreasing fields which is related to the increasing of the Lorentz angle as clearly visible in Figure 7, which in turn produces a larger smearing of the charge distribution at the anode. On the other hand, the behavior of $\mu-\mathrm{TPC}$ is smoother and reaches the minimum resolution values around $1.5 \mathrm{kV} / \mathrm{cm}$. The optimized resolution after the combination of the two methods is obtained for a drift field lying between $1.5 \mathrm{kV} / \mathrm{cm}$ and $2 \mathrm{kV} / \mathrm{cm}$. In this range, we can keep the resolution stable in the full working region.

\section{Applications}

The GEM technology has a large field of applications due to its robustness, workability and low cost with respect to other technologies. In addition, it can be used to cover large area and it can be shaped to the required form. The development of the $\mu-$ TPC reconstruction method allows to obtain spatial resolutions values in high magnetic field never reached so far. This enlarges the field of applications making GEM detector suitable to work in high energy physics experiments in presence of intense field. In this perspective, the Cylindrical Gas Electron Multiplier Inner Tracker (CGEM-IT) project has been developed, which consists in the design and construction of a cylindrical GEM detector as inner tracker for the BESIII experiment [5]. The CGEM-IT is made up of three layers of cylindrical triple-GEM. The first cylindrical 
triple-GEM belongs to KLOE-2 experiment [6], from which we inherited the construction procedure. In order to satisfy the BESIII requirements on spatial resolution and material budget, several improvements were adopted. Rohacell 31, instead of Honeycomb, is used as support for anode and cathode and the permanglass rings located only outside the active area allow to minimize the material budget. CGEM-IT will be the first cylindrical GEM detector with analog readout inside a magnetic field, with simultaneous measurements of deposited charge and time of signal arrival. A dedicated ASIC has being developed: the Torino Integrated GEM Electronics for Readout (TIGER) [7], while a jagged strips anode configuration is adopted to reduce the inter-strips capacitance [8]. The CGEMIT project is funded by the European Commission in the call H2020-MSCA-RISE-2014, and the detector will be installed during the Summer 2018.

\section{CONCLUSION}

Triple-GEM planar chamber have been studied in detail under several working setting (HV, drift fields) and configurations (two different gas mixtures and $3 / 5 \mathrm{~mm}$ drift gaps). A stable spatial resolution as a function of track incident angle and/or in high magnetic field can be achieved only if $\mu-$ TPC method is adopted and combined with charge centroid. A flat distribution of the order of $130 \mu \mathrm{m}$ is expected. Additional beam test data taking and studies are planned in order to finalize and improve the combined $\mu-\mathrm{TPC}$ - charge centroid code to reconstruct the position.

\section{REFERENCES}

[1] F. Sauli, Nucl. Instr. and Meth. A 386, 531 (1997).

[2] J. Benlloch et al., Nucl. Instr. and Meth. A 419, 410 (1998).

[3] M. Iodice, Journal of Instrumentation 9, C01017 (2014).

[4] http://sba.web.cern.ch/sba/BeamsAndAreas/resultbeam.asp?beamline= $\mathrm{H} 4$.

[5] M. Ablikim et al., Nucl. Instr. and Meth. A 614, 345 (2010).

[6] A. Balla et al., Journal of Instrumentation 9, C01014 (2014).

[7] M. Da Rocha Rolo et al., arXiv:1706.02267 (2017).

[8] I. Garzia et al., PoS TIPP2014 292 (2014). 